\title{
Magnetic Interactions in the Geometrically Frustrated Triangular Lattice Antiferromagnet $\mathrm{CuFeO}_{2}$
}

\author{
F. Ye, ${ }^{1}$ J. A. Fernandez-Baca, ${ }^{1,2}$ R. S. Fishman, ${ }^{3}$ Y. Ren, ${ }^{4}$ H. J. Kang,,${ }^{5,6}$ Y. Qiu, ${ }^{5,6}$ and T. Kimura ${ }^{7}$ \\ ${ }^{1}$ Neutron Scattering Science Division, Oak Ridge National Laboratory, Oak Ridge, Tennessee 37831-6393 \\ ${ }^{2}$ Department of Physics and Astronomy, The University of Tennessee, Knoxville, Tennessee 37996-1200 \\ ${ }^{3}$ Materials Science and Technology Division, Oak Ridge National Laboratory, Oak Ridge, Tennessee 37831-6032 \\ ${ }^{4}$ Experimental Facilities Division, Argonne National Laboratory, Argonne, Illinois 60439 \\ ${ }^{5}$ NIST Center for Neutron Research, National Institute of Standards and Technology, Gaithersburg, Maryland 20899-8562 \\ ${ }^{6}$ Department of Materials Science and Engineering, \\ University of Maryland, College Park, Maryland 20742 \\ ${ }^{7}$ Division of Materials Physics, Graduate School of Engineering Science, \\ Osaka University, Toyonaka, Osaka 560-8531, Japan
}

(Dated: January 25, 2020)

\begin{abstract}
The spin-wave excitations of the geometrically frustrated triangular lattice antiferromagnet (TLA) $\mathrm{CuFeO}_{2}$ have been measured using high resolution inelastic neutron scattering. Antiferromagnetic interactions up to third nearest neighbors in the $a b$ plane $\left(J_{1}, J_{2}, J_{3}\right.$, with $J_{2} / J_{1} \approx 0.44$ and $J_{3} / J_{1} \approx$ $0.57)$, as well as out-of-plane coupling $\left(J_{z}\right.$, with $\left.J_{z} / J_{1} \approx 0.29\right)$ are required to describe the spinwave dispersion relations, indicating a three-dimensional character of the magnetic interactions. Two energy dips in the spin-wave dispersion occur at the incommensurate wavevectors associated with multiferroic phase, and can be interpreted as dynamic precursors to the magnetoelectric behavior in this system.

PACS numbers: 75.30.Ds, 75.50.Ee, 61.12.-q
\end{abstract}

Geometrically frustrated magnetic systems have received considerable attention in recent years due to the presence of extraordinary magnetic properties $[1,2,3,4]$. The delafossite $\mathrm{CuFeO}_{2}$ is of particular interest because of the discovery of multiferroic phenomena with either the application of a magnetic field or the substitution of $\mathrm{Fe}^{3+}$ with nonmagnetic $\mathrm{Al}^{3+}$ ions $[5,6,6,7,8]$. As a model material of triangular lattice antiferromagnet (TLA), $\mathrm{CuFeO}_{2}$ forms an Ising-like 4-sublattice ( $\left.\uparrow \downarrow \downarrow\right)$ antiferromagnetic order at low temperature, with spin moment pointing along the $c$ axis [Figs. 1(b) and 1(c)]. This is contrary to other TLA where the three spins align at $120^{\circ}$ from each other in the basal plane [9]. Despite the Heisenberg spin of the orbital singlet of $\mathrm{Fe}^{3+}$ magnetic ions $(L=0, S=5 / 2)$, the metamagnetic transition with magnetic field up to $60 \mathrm{~T}$ [10] and the successive thermal-induced magnetic transition [11] are well explained with the two-dimensional (2D) Ising model, where the $\uparrow \uparrow \downarrow \downarrow$ spin structure is stabilized via the long range magnetic interactions [12]. Recent results suggest that the exchange interaction between adjacent hexagon planes in $\mathrm{CuFeO}_{2}$ could be equally important as the coupling within the planes in this seeming 2D system [13]. In this Letter, we report high resolution inelastic neutron scattering (INS) results of the elementary magnetic excitations in order to clarify the nature of the magnetic interactions in this TLA. We found that magnetic exchange interactions up to the third nearest neighbor (NN) have to be included in order to characterize the dispersion relations in the basal plane. We also found dispersive excitations perpendicular to the hexagonal plane indicative of a substantial interlayer coupling. Most remarkably, we found that the spin-wave dispersion has two dips at the wavevectors associated with the multiferroic phase. Our observations reveal that the quasi-Ising like spin order results from the delicate balance between competing interactions. The static magnetic order is sensitive to external perturbations (magnetic field or impurity), and could be transformed into a noncollinear spin structure that is intimately related to the polar state.

A single crystal $\mathrm{CuFeO}_{2}$ (mass $4.5 \mathrm{~g}$ ) was grown by the floating zone technique, and additional small crystals were crushed to powder for preliminary measurements. The INS measurements were performed at the Disk Chopper Spectrometer and at the cold neutron triple-axis spectrometer SPINS at the NIST Center for Neutron Research. The Disk Chopper Spectrometer measurements on the powder specimen revealed a magnetic excitation bandwidth between $0.9 \mathrm{meV}$ and $3.2 \mathrm{meV}$ [Fig. 1(e)]. We therefore constrained our triple-axis measurements to the energy transfer $E<3.9 \mathrm{meV}$. Although the crystal undergoes a lattice distortion from hexagonal $R \overline{3} m$ to a lower monoclinic $C 2 / m$ symmetry at low $T[14,15]$, we use the hexagonal notation throughout this paper with lattice parameter $a=b=3.03 \AA$ and $c=17.17 \AA$ for the sake of simplicity. The crystal was aligned in the (HHL) scattering plane. At SPINS vertically focused pyrolytic graphite $(\mathrm{PG})$ crystals were used as the monochromator, and the final neutron energy was fixed to $E_{f}=3.7 \mathrm{meV}$. A $\mathrm{BeO}$ filter is placed in the scattered beam to remove the higher order wavelength contaminations. Horizontal collimations of open-80'-sample-80'-open were employed.

Given the spin structure shown in Figs. 1(b) and 1(c), the magnetic Bragg peaks appear at $(0.25,0.25,3 / 2)$ and 

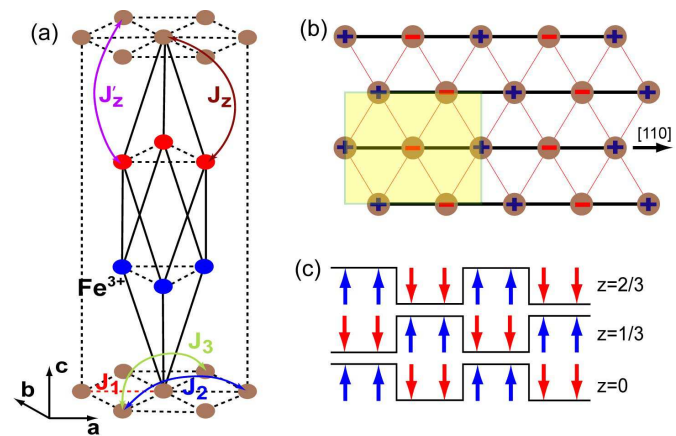

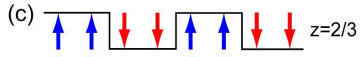

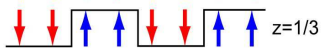

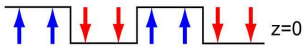
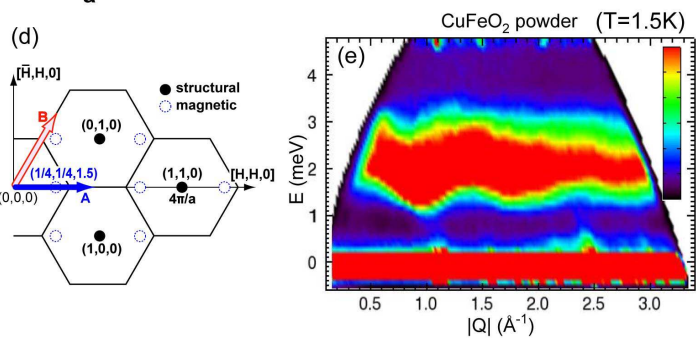

FIG. 1: (Color online) (a) Schematics of the crystal structure and the relevant magnetic exchange interactions between neighboring $\mathrm{Fe}^{3+}$ ions. (b) The ground state spin configuration in the hexagonal plane. The magnetic unit cell is shown by the (yellow) shaded area. (c) The $\uparrow \uparrow \downarrow \downarrow$ spin structures are coupled antiferromagnetically along the $c$ axis. (d) Schematic diagram of the $(H, K, 0)$ scattering plane in reciprocal space. The structural Bragg peaks are represented in solid symbols, the magnetic Bragg peaks displayed in open symbols are projections into that scattering plane. (e) Intensity contour map of the inelastic measured using the time-of-flight Disk Chopper Spectrometer on a powder specimen (mass $\approx 10 \mathrm{~g}$ ), with an incident neutron wavelength of $3.6 \AA$.

equivalent positions. We first studied the magnetic excitations within the hexagonal plane along the $[H, H, 3 / 2]$ direction [the solid arrow in Fig. 1(d)]. Figure 2(a) displays representative energy scans at constant wavevector transfer $\vec{Q}_{\mathrm{s}}$. The scattering profiles are highly structured. In all cases, we identified the lower lying excitation as the spin-wave branch in the $[H, H, 3 / 2]$ direction, while the peaks at higher energies originate from unintended scans along the open arrow shown in Fig. 1(d) due to the crystal hexagonal domains [16]. We notice that the dispersion does not have a minimum at the magnetic Bragg point $(0.25,0.25,3 / 2)$. Instead, two minima appear at symmetric positions at $\vec{Q}_{m 1}=(0.21,0.21,3 / 2)$ and $\vec{Q}_{m 2}=$ $(0.29,0.29,3 / 2)$, with a energy gap $\Delta=0.9 \mathrm{meV}$, in agreement with the data reported by Terada et al. [17]. The scattering profiles along the $[0,0, L]$ direction, on the other hand, show much cleaner features due to the absence of twinned domains. The magnetic excitations along this direction are clearly dispersive, ranging in energy from around $1.3 \mathrm{meV}$ at the zone center to $2.5 \mathrm{meV}$ at the zone boundary. This indicates that the previous treatment of $\mathrm{CuFeO}_{2}$ as a 2D TLA is an oversimplification.
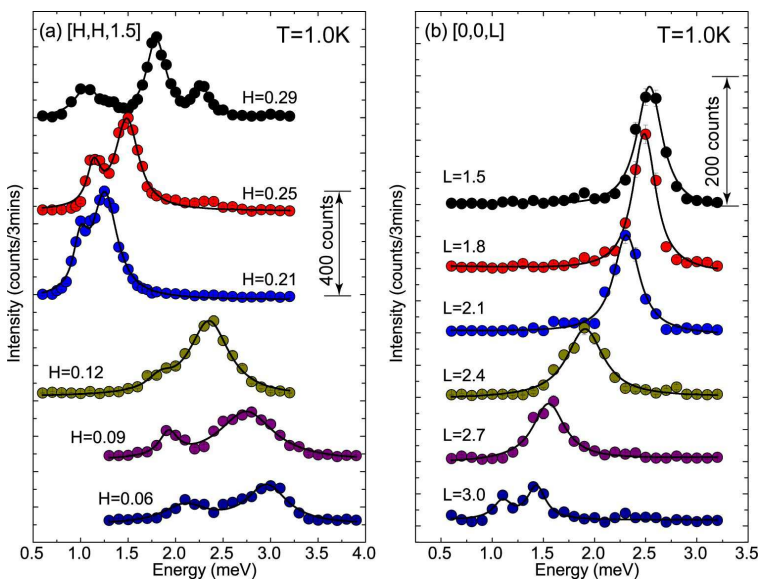

FIG. 2: (Color online) Representative spin-wave excitation scans at $T=1.0 \mathrm{~K}$. Constant wavevector $(\vec{Q})$ scans along (a) the $[H, H, 3 / 2]$ direction and (b) the $[0,0, L]$ direction. Solid lines are Lorentzian fits. Data are shifted for clarity.

In order to obtain an expression of the dispersion relation for $\mathrm{CuFeO}_{2}$, we start with the generic Heisenberg Hamiltonian:

$$
H=-\frac{1}{2} \sum_{i, j} J_{i, j}^{\|} \overrightarrow{S_{i}} \cdot \overrightarrow{S_{j}}-\frac{1}{2} \sum_{i, j} J_{i, j}^{\perp} \vec{S}_{i} \cdot \overrightarrow{S_{j}}-D \sum_{i} S_{i z}^{2},
$$

where $\sum_{i, j}$ indicates summation over pairs of spins. $J_{i, j}^{\|}$ is the in-plane exchange interactions $\left(J_{1}, J_{2}, J_{3}\right.$ and higher order NN coupling as defined in Fig. 1), $J_{i, j}^{\perp}$ is the interactions between adjacent planes $\left(J_{z}\right.$ and $\left.J_{z}^{\prime}\right)$ [18]. $D$ is the single-ion anisotropy. Using a Holstein-Primakoff transformation and a $1 / S$ expansion, we obtain the analytic form of the spin-wave dispersion relation:

$$
\varepsilon^{2}(\mathbf{k})=A^{2}-B^{2} \pm\left[\left(C^{2}-C^{* 2}\right)^{2}+4\left|A C-B C^{*}\right|^{2}\right]^{1 / 2},
$$

where

$$
\begin{aligned}
A= & 2 S\left[D-J_{1}+J_{2}\left(1-\cos k_{y} \sqrt{3} a\right)-J_{3}\left(1+\cos 2 k_{x} a\right)\right. \\
& \left.-J_{z}+2 J_{z}^{\prime}\left(1-\cos k_{z} d \cos k_{x} a\right)\right] \\
B= & -2 S\left[\left(J_{1}+2 J_{3} \cos k_{y} \sqrt{3} a\right) \cos k_{x} a+J_{z} \cos k_{z} d\right] \\
C= & -2 S \cos \left(k_{y} \sqrt{3} a / 2\right)\left[J_{1} e^{i k_{x} a / 2}+J_{2} e^{-3 i k_{x} a / 2}\right] \\
& -4 S J_{z}^{\prime} \cos k_{z} d \cos \left(k_{y} \sqrt{3} a / 2\right) e^{-i k_{x} a / 2} .
\end{aligned}
$$

Here, $k_{x}, k_{y}, k_{z}$ are the wavevectors along the [110], [110], and [001] directions respectively, and $d=c / 3$ is the spacing between adjacent hexagonal planes.

Along the high symmetry [001] direction, the lower branch of the spin-wave dispersion relation described in Eqn. (2) reduces to the much simpler form:

$$
\begin{aligned}
\varepsilon^{2}\left(k_{z}\right)= & 4 S^{2}[(D-G)(D-G+F)+ \\
& \left.F G \cos k_{z} d-G^{2} \cos ^{2} k_{z} d\right],
\end{aligned}
$$

where $F=2\left(J_{2}-2 J_{3}\right)$ and $G=J_{z}-2 J_{z}^{\prime}$. The bandwidth of $\varepsilon^{2}\left(k_{z}\right)$ is mainly controlled by the out-of-plane 


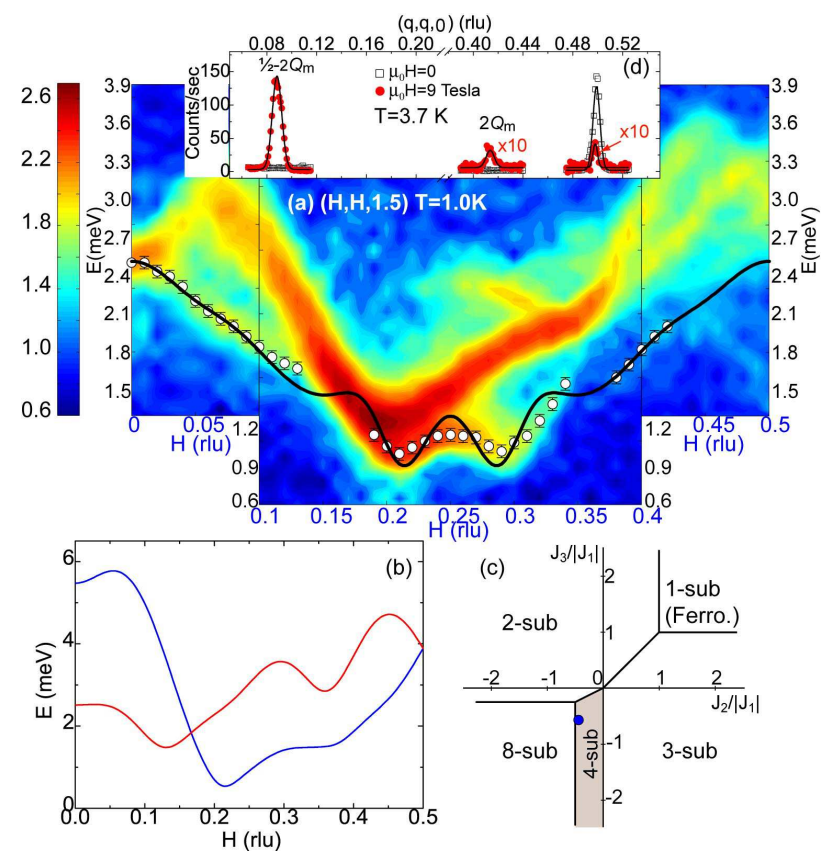

FIG. 3: (Color online) (a) Spin-wave dispersion along the $[H, H, 3 / 2]$ direction. Points are experimental data and the solid line denotes a fit to the model described in the text. Only the data from the lowest branch are included in the analysis. The error bars represent one standard deviation. (b) Predicted dispersion relations along the open arrow in Fig. 1(d) (contribution from the twinning domains). (c) Phase diagram from Ref. [12]. The solid symbol is the value obtained from our measurements. (d) The structural superlattice peaks appear at $\vec{Q}_{L}=2 \vec{Q}_{m}$ and $\vec{Q}_{L}=1 / 2-2 \vec{Q}_{m}$ (due to exchange striction) when the incommensurate spin order occurs at $\mu_{0} H=9$ Tesla.

interactions $J_{z}$ and $J_{z}^{\prime}\left[\right.$ i.e. $\left.\varepsilon^{2}(1.5)-\varepsilon^{2}(3.0)=-8 S^{2} F G\right]$, while the single-ion anisotropy term $D$ is determined by fitting the experimental data to the reduced dispersion relation Eq. (3). We obtained $D S=0.17 \mathrm{meV}$, which is larger than the estimated value of $D S=0.05 \mathrm{meV}$ from magnetization measurement [13]. The solid lines in Fig. 3(a) and Fig. 4 represent the global fits to the observed spin-wave energies along $[H, H, 3 / 2],[0,0, L]$ and $[q, q, L]$ (with $q=0.21$ and 0.25 ) using the model described above. Long-range antiferromagnetic interactions (up to $\left.J_{3}\right)$ are found $\left(J_{1} S=-1.14 \mathrm{meV}, J_{2} S=\right.$ $-0.50 \mathrm{meV}$, and $J_{3} S=-0.65 \mathrm{meV}$ ) to satisfactorily describe the in-plane dispersion relation [19]. The inclusion of higher order interactions $\left(J_{4} S\right)$ in our analysis did not improve the fits. Only one NN exchange interaction $\left(J_{z} S=-0.33 \mathrm{meV}\right)$ is necessary to characterize the spin-wave dispersion out of the basal plane, the addition of a second out-of-plane exchange interaction also yields a small value $\left(J_{z}^{\prime} S=0.01 \mathrm{meV}\right)$ with no significant improvement of the fit.

We now compare our fitting parameters with the earlier theoretical calculations. The phase diagram of the
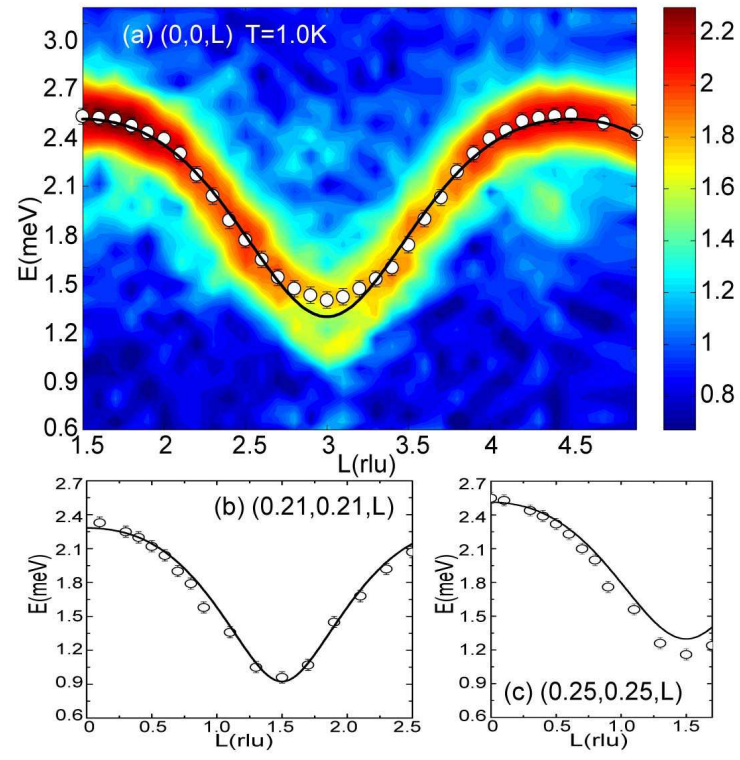

FIG. 4: (Color online) Spin-wave dispersions along the (a) $[0,0, L]$, (b) $[0.21,0.21, L]$, and (c) $[0.25,0.25, L]$ direction. Points are experimental data and the solid line are fits to the model described in the text. Only the data at the lowest branch are presented in (b) and (c).

2D Ising spin TLA has been investigated by Takagi and Mekata using Monte Carlo simulations [12]. They found that the collinear 4-sublattice structure is only stable with fairly large $J_{2}$ and $J_{3}$ contributions. The $\uparrow \uparrow \downarrow \downarrow$ phase is bound within a narrow range of $-0.5<J_{2} /\left|J_{1}\right|<0$ and $J_{3} / J_{2}<0.5$ [the shaded area in Fig. 3(c)]. Our result, with $J_{2} / J_{1}=0.44$ and $J_{3} / J_{1}=0.57$, is positioned within this shaded region near the boundary where the 4sublattice state coexists with the 8-sublattice state [12]. The phase diagram is not affected by the inclusion of the single-ion anisotropy $D$, as this term uniformly shifts the energy at each spin site. Similarly, this phase diagram remains unchanged with respect to the NN out-of-plane exchange coupling $J_{z}$, since the total energy stays invariant regardless of the detailed in-plane magnetic structure, providing there is a negligible $J_{z}^{\prime}$. The agreement between our estimation of the in-plane exchange constants and those predicted by Takagi and Mekata implies that the mean field approach can effectively explain the emergence of the long-range magnetic order.

Another important result is the observation of two energy dips at $\vec{Q}_{m 1}$ and $\vec{Q}_{m 2}$ in symmetric positions around the commensurate magnetic Bragg point $\tau=$ $(0.25,0.25,3 / 2)$ characteristic of the 4 -sublattice phase. Remarkably these are precisely the same wavevectors associated with the incommensurate magnetic order of $\mathrm{CuFeO}_{2}$ under a magnetic field [20], and are dynamic precursors to the multiferroic phase. By decreasing the single-ion anisotropy $D$ without modifying the exchange constant $J_{i}$ 's, one can essentially close the spin gap $\Delta$, 
while the characteristic $\vec{Q}_{m}$ related to the energy minimum remain unchanged. In any antiferromagnet, the spin-wave modes are linearly split in a magnetic field and a critical value of $H_{c}(T)=\Delta / g \mu_{B}$ will destroy the local stability of the antiferromagnetic state. For $\Delta=0.9 \mathrm{meV}$ this critical value is 7.7 Tesla, which is in agreement with the experimental observation that incommensurate magnetic order appears for $\mu_{0} H>7.0$ Tesla [5]. The softening of the magnetic dynamics achieved by applying a magnetic field reveals a close connection between the breakdown of the $\uparrow \uparrow \downarrow \downarrow$ spin structure and the formation of a new type of magnetic order. The local stability of the antiferromagnetic state can also be destroyed by chemical substitution. A polar state has been recently discovered in the doped $\mathrm{CuFe}_{1-\mathrm{x}} \mathrm{Al}_{\mathrm{x}} \mathrm{O}_{2}$ in zero magnetic field possessing an incommensurate spin structure with the same $\vec{Q}_{m}[7,[8]$.

There remain open questions in this TLA system. For instance, why are the exchange interactions up to $J_{3}$ required to stabilize the collinear spin structure? Although the slight increase in bonding angle of Fe-O-Fe along the [110] direction [Fig. 1(b)] below the Néel temperature might explain a dominant antiferromagnetic interaction $J_{1}$, there is no obvious justification for the existence of comparable AF interactions between higher order neighboring spins. Second, the microscopic origin of the anisotropy gap $\Delta$ is yet to be understood. One would expect the magnetic properties should be isotropic since $\mathrm{Fe}^{3+}$ is an orbital singlet. One possible explanation might be related to the lattice distortion accompanied with the magnetic order which induces an anisotropy between the Fe-3 $d$ and $\mathrm{O}-2 p$ hybridization as well as the charge transfer between them [21]. Finally, the excitation data are not completely characterized by the Heisenberg model described in Eq. (1). Figure 3(b) shows the expected dispersion curves from the twinned magnetic domains. Only some features agree with the experimental data. Asymmetric dispersion curves appear with local minima located at $\vec{Q}=(0.22,0.22,3 / 2)$ and $(0.36,0.36,3 / 2)$. However, the magnetic gap and overall energy bandwidth do not agree with the observations.

In summary, the spin-wave spectrum of the geometrically frustrated TLA $\mathrm{CuFeO}_{2}$ has been mapped out using INS. We observed a considerably dispersive excitation along the $c$ axis, which reflects the 3D character of the magnetic interactions. We have also determined the relevant spin Hamiltonian parameters, confirming that the next NN and the third NN interactions are required to explain the spin dynamics. Finally, the local minimum of the dispersion curves reveal the dynamic precursory to the multiferroic phase.

We are grateful to D. I. Khomskii, S. Mitsuda and N. Terada for useful discussions. ORNL is supported by U.S. DOE under Contract No. DE-AC05-00OR22725 with UT/Battelle LLC.This work utilized facilities supported in part by the National Science Foundation under Agreement No. DMR-0454672.

[1] A. Ramirez et al., Nature (London) 399, 333 (1999).

[2] T. Kimura et al., Nature (London) 426, 55 (2003).

[3] N. Hur et al., Nature (London) 429, 392 (2004).

[4] T. Lottermoser et al., Nature 430, 541 (2004).

[5] T. Kimura, J. C. Lashley, and A. P. Ramirez, Phys. Rev. B 73, 220401(R) (2006).

[6] S. Seki, et al., Phys. Rev. B 75, 100403(R) (2007).

[7] T. Nakajima, et al., J. Phys. Soc. Jpn. 76, 043709 (2007).

[8] S. Kanetsuki, et al., J. Phys.:Condens. Matter 19, 145244 (2007).

[9] M. F. Collins and O. A. Petrenko, Can. J. Phys. 75, 605 (1997). H. Kawamura, J. Phys.: Condens. Matter 10, 4707 (1998).

[10] Y. Ajiro et al., Physica B 201, 71 (1994). S. Mitsuda, et al., J. Phys. Chem. of Solids 60, 1249 (1999).

[11] S. Mitsuda, N. Kasahara, T. Uno, and M. Mase, J. Phys. Soc. Jpn. 67, 4026 (1998).

[12] T. Takagi and M. Mekata, J. Phys. Soc. Jpn. 64, 4609 (1995).

[13] O. A. Petrenko, et al., J. Phys.: Condens. Matter, 17, 2741, (2005).

[14] F. Ye, et al., Phys. Rev. B 73, 220404(R) (2006).

[15] N. Terada, et al., J. Phys. Soc. Jpn. 75, 023602 (2006).

[16] The single crystal has three equally populated magnetic domains in the basal plane. We simultaneously probed the excitations from the twinning domains which are $60^{\circ}$ apart in the reciprocal space. The stronger scattering intensities at higher energies are consistent with these contributions.

[17] N. Terada et al., J. Mag. Mag. Mat. 272-276, e997 (2004). N. Terada, et al., J. Phys: Condens. Matter, 19, 145241 (2007).

[18] We use simplified out-of-plane interactions where Fe ions couple directly to the ones at the top and the bottom. This is slightly different from the actual stacking pattern shown in Fig. 1(a).

[19] A known lattice distortion ( $\Delta a / a<0.4 \%$, Ref. [14, 15]) may cause a small change in the exchange constants along one direction that stabilizes the 4-sublattice phase along that direction. It is unlikely, that this will significantly modify the spin-wave dispersion described in this paper.

[20] Using synchrotron x-ray (Ref. 14]) and neutron scattering [Fig. 3(d)], we found that an incommensurate lattice modulation with $\vec{Q}_{L}=(0.91,0.91,0)$ and $(0.09,0.09,0)$ appears in the multiferroic phase of $\mathrm{CuFeO}_{2}$. The relationship between $\vec{Q}_{L}$ and $\vec{Q}_{m}$ for the corresponding incommensurate magnetic structure is given by: $\vec{Q}_{L}=$ $(1 / 2,1 / 2,0) \pm\left(2 q_{m}, 2 q_{m}, 0\right)$ and $\vec{Q}_{m}=\left(q_{m}, q_{m}, 3 / 2\right)$, where $q_{m}=0.205$. The two-to-one ratio of the lattice and magnetic structure is due to the strong magnetoelastic coupling.

[21] N. Terada et al., Phys. Rev. B 75, 224411 (2007). 